http://jmscr.igmpublication.org/home/ ISSN (e)-2347-176x ISSN (p) 2455-0450 crossref DOI: https://dx.doi.org/10.18535/jmscr/v8i7.23

\title{
Early Diagnosis of Congenital Insensitivity to Pain with Anhidrosis in an infant- a Case Report
}

\author{
Authors \\ Dr Pai Ashutosh A., Dr Deshmukh Isha, Dr Parween Shaista \\ Department of Paediatrics, Grant Government Medical College and JJ Hospital \\ *Corresponding Author \\ Dr Pai Ashutosh A
}

\begin{abstract}
Congenital insensitivity to pain with anhidrosis is extremely rare form of Hereditary neuropathy. We report this 4 month old male child noticed to have fever since 10 days. Child had history of multiple visits for fever. On examination, weak cry, good suck, dry skin, bilateral ptosis, hypotonic, unresponsive to noxious stimuli and temperature sensations with normal touch sensation, power and Deep Tendon Reflexes. All relevant blood investigations were inconclusive. Child was treated with multiple antibiotics, with no effect. Based upon the presenting clinical features and investigations, genetic testing for CIPA was done. Likely Pathogenic variant of gene identified.

Keywords: CIPA; fever; hereditary; neuropathy.
\end{abstract}

\section{Introduction}

Congenital insensitivity to pain with anhidrosis (CIPA, hereditary sensory and autonomic neuropathy type IV) is an autosomal recessive disorder with only a handful of cases reported from India ${ }^{1}$. Three clinical findings define the syndrome: absent reaction to noxious stimuli, inability to sweat, and developmental delay. CIPA is the clinical presentation of a loss of function mutation in the Neurotrophic tyrosine kinase receptor type 1 (NTRK1) gene, located on chromosome number 1 which encodes for one of the receptors for the nerve growth factor namely TrkA. Herein we report a rare case of CIPA diagnosed at an earlier than usual age highlighting importance of keeping high index of suspicion for such diagnoses.

\section{Case Report}

4 month old male child, first by birth order, born of third degree consanguinity, noticed to have fever since 10 days. Fever was documented as high grade (100*F to $102 * \mathrm{~F})$, continuous type, not associated with chills, rigors or rash, responding to antipyretics and tepid water sponging, with no clinically identifiable focus of infection. Baby was born full term by Lower Segment Caesarean Section in view of nonprogress of labour, baby cried immediately after delivery, did not require artificial respiration. Baby had weak pitched cry and hence was admitted to Neonatal ICU for observation. Septic screen was done on day 1 and repeated on day 3 which was normal. Lumbar puncture was done which ruled out meningitis. Child had no seizures during the NICU stay. Childs cry did not improve 
but was taking breastfeeding well. Child would remain floppy during rest. Baby was treated as clinical meningitis with antibiotics for 14 days. He showed partial improvement in cry with adequate weight gain hence was discharged. Prior to current admission, child has history of repeated OPD visits and 2 previous admissions for recurrent non resolving fever. We also noticed that the child had not cried during venepuncture or immunisation in any of his previous visits. Parents noticed that the child has had no sweating anywhere on the body till date. No family history of similar complaints. Child has not achieved head control but has achieved roll over and social smile. On general examination pallor was present, central bald patch seen on the scalp, dry rough skin, drooping eyelids bilaterally. There were no hypo pigmented patches or thickened, palpable nerves. Motor examination showed generalised hypotonia and wasting. Power was more than $3 / 5$ in all 4 limbs. Deep tendon reflexes were normal. Sensory examination showed absent reaction to noxious stimuli tested with sharp pin prick all over the body associated with global loss of temperature sensations tested by placing hot and cold objects close to the skin all over the body, but fine touch was preserved. Among neonatal reflexes, Asymmetric tonic neck reflex persisted. Lacrimation was normal. Cranial nerve examination was normal. Ciliospinal reflex was normal. Complete hemogram revealed iron deficiency anaemia with normal WBC and platelet counts. Repeated Blood cultures, Urine cultures and CSF cultures isolated no organism. Thyroid profile was normal. $2 \mathrm{D}$ Echo showed no evidence of infective endocarditis. Peripheral smear has no abnormal cell types. Brain MRI was normal .Child was treated with multiple antibiotics, antivirals, antifungals and antimalarial but with no effect on fever. A referral to paediatric neurologist was made. Nerve conduction velocity testing was done. For motor nerves the median, ulnar, tibial and peroneal nerves were tested for the amplitude, latency, duration and velocity of conduction of impulses across the nerves which were normal.
For sensory nerve testing the median, ulnar and sural nerves were tested for the same parameters which were normal. Skin biopsy could not be done as it was not feasible in such a small child as opined by paediatric neurologist. Based upon the presenting clinical features and investigations, presumptive diagnosis of CIPA was made. Clinical exome sequencing was done by sequencing protein coding regions in genes associated with inherited diseases at a mean coverage of 80-100X in the target region. An exon was considered to have full coverage if all coding bases plus three nucleotides of flanking sequence on either side are covered at $20 \mathrm{X}$. "Likely Pathogenic" homozygous missense mutated variant of the NTRK1 gene (p.Arg648Cys) in exon 14 of the gene was identified. (American College of Medical Genetics guidelines)The observed variant is not present in the 1000 Genomes database but is reported in gnom $\mathrm{AD}$ database with minor allele frequency of $0.0016 \%$. The variant is present in Protein tyrosine kinase domain of NTRK1_HUMAN protein. The variant is present in the hotspot region with 2 other variants (p.Arg649Gln, p.Val647Leu) .No other pathogenic variants in genes unrelated to the patients phenotype or in genes related on the ACMG recommended secondary list were detected. Genetic counselling of the parents was done for genetic testing and risk assessment for future pregnancies. Parents are yet to be tested at the time of writing this report. Detailed advice was given about special measures to be taken to treat primary manifestations and prevent secondary complications.

\section{Discussion}

Congenital insensitivity to pain with anhidrosis (CIPA) is an autosomal recessive disorder. Half the cases reported have occurred in consanguineous marriages. Apart from the already well-defined genetic transmission of CIPA, there is an infrequent non-Mendelian inheritance characterized by uniparental disomy ${ }^{2}$ 


\section{Clinical presentation of CIPA}

The first signs are defects in thermoregulation due to failure to sweat and recurrent episodes of hyperthermia and unexplained fever that begin in early infancy. Recurrent febrile convulsions are also observed in some affected infants. Anhidrosis is present on the trunk and upper extremities in $100 \%$ of cases and more variable in other areas of the body.While impaired pain perception may not be apparent in early infancy, parents may recall that their infant with CIPA did not cry during venepuncture or immunizations ${ }^{3}$. Tongue ulcers and fingertip biting, the characteristic selfmutilation observed in infants with CIPA, begin when the primary incisors erupt, and can result in a bifid or absent tongue. Biting of the fingers and ulcerated fingertips is common. Bruises, cuts, and burns do not elicit normal reactions and are often unrecognized at the time that they occur. Accidental injuries such as falls or burns lead to multiple scars and can lead to cellulitis in the skin. Orthopaedic problems are one of the most characteristic and serious complications of $\mathrm{CIPA}^{4}$ .Repeated trauma and improper healing can lead to chronic osteomyelitis or Charcot's arthropathy. Decreased pain perception does not spare even cranial nerves and visceral sensation

Assessments of cognitive and adaptive behaviour suggest that many children with CIPA have intellectual disability (or learning disabilities) and severe attention-deficit-hyperactivity disorder (ADHD). Rarely, it also can present with features of impaired immune response, proteinuria, renal failure, anaemia, hyperkeratosis, dry skin, and early primary tooth loss. Death from hyperpyrexia within the first three years of life may occur in $20 \%$ of these cases. Hypotonia is seen frequently in the early years, but strength and tone normalize as the individual gets older; tendon reflexes are normal gastrointestinal dysmotility is mild or absent. Vomiting is not a classic feature. Speech is usually clear. $^{5}$

CIPA has to be differentiated from other hereditary sensory and autonomic neuropathies. The clinical manifestations of CIPA can be similar to those seen in Leprosy, Hypohydrotic

Ectodermal Dysplasia or Lesch Nyhan Syndrome (self-mutilation)

\section{Diagnosis of CIPA}

The diagnosis of CIPA is made clinically. Neurologic examination supports the diagnosis. Painful stimuli fail to evoke either withdrawal or emotional change ${ }^{6}$. Consistent errors are made in distinguishing between hot and cold moist substances. Normal findings are: Touch, vibration and position senses, Motor functions, Deep tendon reflexes and superficial abdominal and cremasteric reflexes. Additional tests supporting the diagnosis of CIPA include- Skin tests demonstrating abnormalities in sweating, Histamine test- An intradermal injection of histamine produces in controls both a local wheal and flare, but in individuals with CIPA only produces a local wheal and no flare. Skin Biopsy on gross examination appears normal. Histologic studies demonstrate normal sweat glands, sebaceous glands, hair follicles, and nerve receptors. Electron microscopic studies reveal lack of innervation of the eccrine sweat glands, blood vessels and erector pilae muscle with complete absence of small-diameter myelinated and unmyelinated nerve fibres. The diagnosis of CIPA is confirmed by identification of biallelic pathogenic variants in NTRK1 gene by Clinical Exome Sequencing .Classification of variants is done as per ACMG guidelines. ${ }^{7}$

\section{Treatment of CIPA}

Treatment is supportive and is best provided by specialists in paediatrics, orthopaedics, dentistry, ophthalmology, and dermatology. The therapeutic approach to CIPA is still evolving and remains controversial. There is no definitive agreement regarding its management, and therapeutic options are restricted to treatment of primary symptoms and prevention of complications. ${ }^{8}$ Monitoring of the body temperature helps to institute timely measures to prevent hyperthermia or hypothermia. Hyperthermia responds to direct cooling in a bath or cooling blanket; hypothermia responds to warming by a blanket as necessary. Bone fractures 
of weight-bearing bones and joints can lead to failure of bone union and hypertrophic osseous callous formation. Hence the goal of orthopaedic management is to prevent severe articular destruction and the need for surgical amputation. Appropriate footwear and periods of non-weightbearing are important in the prevention and early treatment of ulcerating foot lesions Methods used to prevent injuries to the lips, buccal mucosa, tongue, and teeth include tooth extraction, and/or filing (smoothing) of their sharp incisal edges and/or use of a mouth guard, a protective plate of thermoplastic resin $\sim 0.6-0.8 \mathrm{~mm}$ thick. Care for dry eyes, prevention of corneal infection, and daily observation of the ocular surface are crucial for maintaining good visual function. Daily care with a skin moisturizer is recommended to prevent or reduce skin cracking. Interventions for behavioural, developmental and motor delays as well as educational and social support for schoolage children and adolescents are important. ${ }^{9}$

Genetic counselling is the process of providing individuals and families with information on the nature, inheritance, and implications of genetic disorders to help them make informed medical and personal decisions. It is important to deal with genetic risk assessment and the use of family history and genetic testing to clarify genetic status for family members. The optimal time for determination of genetic risk, clarification of carrier status, and discussion of the availability of prenatal testing is before pregnancy. ${ }^{10}$

\section{Conclusion}

In families who have a patient of CIPA, prenatal screening should be done to prevent the birth of another affected child. High index of suspicion is necessary for early diagnosis so that affected children can be protected from orthopaedic complications and accidental injuries by regular visual and dental follow-up. A unique feature of our case is that a diagnosis was made even before any complications could arise and hence timely measures could be taken to effectively prevent them. This case report is especially important to clinicians who are working in countries or with cultures where consanguineous marriages are a common practice as half the reported cases are born of consanguineous marriage.

\section{References}

1. Udayashankar C, Oudeacoumar P, Nath AK. Congenital insensitivity to pain and anhidrosis: a case report from South India. Indian J Dermatol 2007; 57(6):503.

2. G. Tunc,bilek, C. ' Oztekin, A. Kayikc, io glu. "Calcaneal ulcer in a child with congenital insensitivity to pain syndrome" Scandinavian Journal of Plastic and Reconstructive Surgery and Hand Surgery 2005; 39(3):180-183.

3. Miura $\mathrm{Y}$ et al "Complete paternal uniparental disomy for chromosome 1 revealed by mutation analyses of the TRKA (NTRK1) gene encoding a receptor tyrosine kinase for nerve growth factor in a patient with congenital insensitivity to pain with anhidrosis." Hum Genet. 2000; 107:205-9

4. Abdel-Hafez HZ, Awad SM. "Congeintal insensitivity to pain with anhidrosis (CIPA)" Egypt Dermatol Online J. 2007;3: 186-192.

5. Y. Indo, "Molecular basis of congenital insensitivity to pain with anhidrosis (CIPA): mutations and polymorphisms in TRKA (NTRK1) gene encoding the receptor tyrosine kinase for nerve growth factor," Human Mutation 2001; 18(6): 462-471.

6. R. Schwarzkopf, V. Pinsk, Y. Weisel, D. Atar, and Y. Gorzak, "Clinical and genetic aspects of congenital insensitivity to pain with anhidrosis," Harefuah 2005;144(6): 433-437.

7. Indo Y. "Genetics of congenital insensitivity to pain with anhidrosis (CIPA) or hereditary sensory and autonomic neuropathy type IV. Clinical, biologicial and molecular aspects of 
mutations in TRKA (NTRK1) gene encoding the receptor tyrosine kinase for nerve growth factor." Clin Auton Res. 2002; 12 Suppl 1:I20-32.

8. Amano A, Akiyama S, Ikeda M, Morisaki I. "Oral manifestations of hereditary sensory and autonomic neuropathy type IV. Congenital insensitivity to pain with anhidrosis." Oral Surg Oral Med Oral Pathol Oral Radiol Endod. 1998;86:42531.

9. Bar-On E, Weigl D, Parvari R, Katz K, Weitz R, Steinberg T. Congenital insensitivity to pain. "Orthopedic manifestations. Bone Joint" Surg $\mathrm{Br} 2002 ; 84: 252-7$.

10. Toscano E. "Hereditary sensory and autonomic neuropathy type IV." Orphanetencyclopedia. 2003. 